\title{
GGCX variants leading to biallelic deficiency to $\gamma$-carboxylate GRP cause skin laxity in VKCFD1 patients
}

\author{
Suvoshree Ghosh ${ }^{1}$, Katrin Kraus ${ }^{1}$, Arijit Biswas ${ }^{2}$, Jens Müller ${ }^{3}$, Francesco Forin ${ }^{4}$, Heike \\ Singer $^{5}$, Klara Höning ${ }^{6}$, Veit Hornung ${ }^{7}$, Matthias Watzka ${ }^{4}$, Johannes Oldenburg ${ }^{8}$, and \\ Katrin Czogalla-Nitsche ${ }^{9}$ \\ ${ }^{1}$ University Clinic Bonn \\ ${ }^{2}$ Universitatsklinikum Bonn \\ ${ }^{3}$ University Clinic Bonn \\ ${ }^{4}$ University Clinic Bonn \\ ${ }^{5}$ University Clinic Bonn \\ ${ }^{6}$ University Hospital Bonn \\ ${ }^{7}$ Ludwig Maximilians University Munich \\ ${ }^{8}$ University Hospital Bonn Institute for Experimental Hematology and Transfusion \\ Medicine \\ ${ }^{9}$ Institute of Experimental Haematology and Transfusionmedicine
}

April 22, 2021

\begin{abstract}
$\gamma$-Glutamyl carboxylase (GGCX) catalyses $\gamma$-carboxylation of 15 different vitamin K dependent (VKD) proteins. Pathogenic variants in GGCX cause a rare hereditary bleeding disorder called Vitamin K dependent coagulation factor deficiency type 1 (VKCFD1). In addition to bleedings, some VKCFD1 patients develop skin laxity and skeletal dysmorphologies. However, the pathophysiological mechanisms underlying these non-haemorrhagic phenotypes remain elusive. Therefore, we analyzed the effect of 22 GGCX pathogenic variants on $\gamma$-carboxylation of six non-haemostatic VKD proteins (UCMA/GRP, MGP, BGLAP, GAS6, PRGP1, TMG4) in a GGCX-/- HEK293T cell line by a functional ELISA. We observed that biallelic deficiency to $\gamma$-carboxylate Gla-rich protein lead to the development of skin laxity. Markedly reduced level of $\gamma$-carboxylated MGP is crucial but not exclusive for causing facial dysmorphologies. Moreover, we identified the vitamin $\mathrm{K}$ hydroquinone binding site in GGCX in an in silico model by docking studies, which was further validated by functional assays. Variants affecting this site result into loss-of-function or severely diminished ability to $\gamma$-carboxylate VKD proteins and hence are involved in the most severe phenotypes. This genotype-phenotype analysis will help to develop new treatment options for VKCFD1 patients, where individualized therapy with $\gamma$-carboxylated VKD proteins may represent a promising strategy.
\end{abstract}

\section{Original Article}

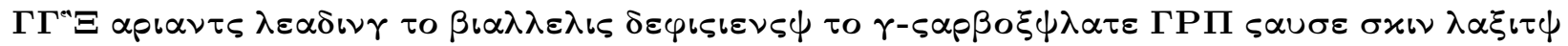

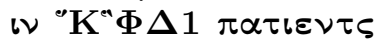

Running head: GRP determines skin laxity

Suvoshree Ghosh ${ }^{1}$, Katrin Kraus ${ }^{1}$, Arijit Biswas ${ }^{1}$, Jens Müller ${ }^{1}$, Francesco Forin ${ }^{1}$, Heike Singer ${ }^{1}$, Klara Höning $^{2}$, Veit Hornung ${ }^{2,3}$, Matthias Watzka ${ }^{1,4}$, Johannes Oldenburg $^{1,4^{*}}$, Katrin J. Czogalla-Nitsche ${ }^{1^{*}}$ 
${ }^{1}$ Institute of Experimental Haematology and Transfusion Medicine, University Clinic, Venusberg-Campus 1,53127

${ }^{2}$ Institute for Clinical Chemistry and Clinical Pharmacology, Unit for Clinical Biochemistry, ,

${ }^{3}$ GeneCenter and Department of Biochemistry, Ludwig-Maximilians-Universität München,

${ }^{4}$ Center for Rare Diseases, University Clinic, Venusberg-Campus 1, 53127

Corresponding authors:

Dr. Johannes Oldenburg

Institute of Experimental Haematology and Transfusion Medicine

University Clinic

Venusberg-Campus 1

53127

E-mail: Johannes.Oldenburg@ukbonn.de

Dr. Katrin Czogalla-Nitsche

Institute of Experimental Haematology and Transfusion Medicine

University Clinic

Venusberg-Campus 1

53127

E-mail: Katrin.Czogalla@ukbonn.de

phone: +49-228-287-16783

Abstract: 195 words

Words main: 3806 words

Tables and Figures: 6

References: 38

Supporting Information: 7 Figures and 3 Tables

\begin{abstract}
$\gamma$-Glutamyl carboxylase $(G G C X)$ catalyses $\gamma$-carboxylation of 15 different vitamin $\mathrm{K}$ dependent (VKD) proteins. Pathogenic variants in $G G C X$ cause a rare hereditary bleeding disorder called Vitamin K dependent coagulation factor deficiency type 1 (VKCFD1). In addition to bleedings, some VKCFD1 patients develop skin laxity and skeletal dysmorphologies. However, the pathophysiological mechanisms underlying these nonhaemorrhagic phenotypes remain elusive. Therefore, we analyzed the effect of 22 GGCX pathogenic variants on $\gamma$-carboxylation of six non-haemostatic VKD proteins (UCMA/GRP, MGP, BGLAP, GAS6, PRGP1, TMG4) in a $G G C X^{-/-}$HEK293T cell line by a functional ELISA. We observed that biallelic deficiency to $\gamma$ carboxylate Gla-rich protein lead to the development of skin laxity. Markedly reduced level of $\gamma$-carboxylated MGP is crucial but not exclusive for causing facial dysmorphologies. Moreover, we identified the vitamin K hydroquinone binding site in GGCX in an in silico model by docking studies, which was further validated by functional assays. Variants affecting this site result into loss-of-function or severely diminished ability to $\gamma$ carboxylate VKD proteins and hence are involved in the most severe phenotypes. This genotype-phenotype analysis will help to develop new treatment options for VKCFD1 patients, where individualized therapy with $\gamma$-carboxylated VKD proteins may represent a promising strategy.
\end{abstract}




\section{Keywords:}

VKCFD1, GGCX, UCMA/GRP, MGP, PXE-like

\section{Introduction}

Vitamin K dependent coagulation factor deficiency type 1 (VKCFD1; OMIM \#277450) is a rare hereditary bleeding disorder caused by pathogenic variations in $\gamma$-Glutamyl carboxylase (GGCX) gene (Watzka et al., 2014). GGCX is an enzyme located in the ER that converts specific glutamic acid residues (Glu) into $\gamma$-carboxyglutamic acid residues (Gla) in vitamin K dependent (VKD) proteins (Rishavy \& Berkner, 2012; Vermeer, 1990). This post-translational modification is essential and renders VKD proteins to their active form. VKCFD1 patients are diagnosed with bleeding symptoms caused by decreased activity of VKD clotting factors. Seven out of 15 VKD proteins have functions in the coagulation cascade including coagulation factor II (FII), VII (FVII), IX (FIX), X (FX), Proteins C (PC), S and Z (Kurachi \& Davie, 1982; Leytus, Foster, Kurachi, \& Davie, 1986; Nelsestuen, 1999; Stenflo, Fernlund, Egan, \& Roepstorff, 1974). In addition to the haemorrhagic phenotype (Brenner et al., 1998; Darghouth et al., 2006; Spronk, Farah, Buchanan, Vermeer, \& Soute, 2000), several VKCFD1 patients were reported to develop additional non-haemorrhagic phenotypes such as Pseudoxanthoma elasticum (PXE)-like syndrome causing skin hyperlaxity and folds, cardiac abnormalities, and osseous phenotypes such as midfacial hypoplasia or Keutel-like syndrome which is characterized by brachytelephalangy of the fingers and facial dysmorphologies (De Vilder, Eva Y G, Debacker, \& Vanakker, 2017 Jan 25). These additional phenotypes are most likely developed due to the under-carboxylation of the other non-haemostatic VKD proteins which include matrix Gla protein (MGP), upper zone of growth plate and cartilage matrix associated protein (UCMA/GRP), osteocalcin (BGLAP), proline-rich Gla proteins (PRGPs) 1 and 2, and transmembrane Gla proteins (TMGs) 3 and 4, and growth arrest specific 6 (GAS6) (Kulman, Harris, Xie, \& Davie, 2001; Manfioletti, Brancolini, Avanzi, \& Schneider, 1993; Poser, Esch, Ling, \& Price, 1980; Price, Fraser, \& Metz-Virca, 1987; Viegas et al., 2009; Viegas et al., 2015). These proteins have diverse functions where BGLAP, MGP, and GRP have role in the regulation of physiological calcification in bone or soft tissues (Lacombe \& Ferron, 2015; Willems, Vermeer, Reutelingsperger, \& Schurgers, 2014) . GAS6 is a ligand of TAM receptor (Tyro, Axl, and Mer) and has function in cell signaling f.e. proliferation and platelet aggregation (Berkner \& Runge, 2004). The function of the four VKD transmembrane proteins PRGP1 and 2, TMG3 and 4 is not yet clear.

The mechanism of $G G C X$ variants leading to the non-haemorrhagic phenotypes is unknown. Previous studies have evaluated the effect of few selected GGCX variants on $\gamma$-carboxylation of either MGP or BGLAP among all non-haemostatic proteins (Tie et al., 2016; Watzka et al., 2014). However, whether the undercarboxylation of other known VKD proteins contributes to the non-haemorrhagic phenotypes remains elusive. VKCFD1 patients are mainly treated with high dose of vitamin $\mathrm{K}$ for correcting the clotting factor activities, but it is unclear whether life-long treatment with vitamin $\mathrm{K}$ is sufficient to rescue non-haemorrhagic phenotypes. Therefore, to devise new treatment strategies in the future, first we have to determine which undercarboxylated VKD protein/s are leading to specific phenotypes.

In the present study, an extensive genotype-phenotype analysis was performed by evaluating the effect of 22 reported GGCX variants on $\gamma$-carboxylation of six non-haemostatic VKD proteins in a $G G C X^{-/}$ HEK293T cell line. We identified that undercarboxylated GRP causes the PXE-like phenotype by correlating the $\gamma$-carboxylation status from our analysis with the existing patient data. The knowledge of causative VKD protein will help to treat VKCFD1 patients in the future with personalized medicines. Additionally, we performed a $\mathrm{KH}_{2}$ docking on a GGCX in silicomodel, on which we have identified the $\mathrm{KH}_{2}$ binding site. Pathogenic variants affecting this site showed either loss-of-function or extreme reduced ability to $\gamma$-carboxylate VKD proteins.

\section{Methods}

\section{Construction of human GGCX/VKD protein expression vectors}

The cDNAs of VKD proteins (UCMA/GRP, MGP, BGLAP, PRGP1, TMG4, GAS6) together with a c-myc 
tag at the C-terminus were cloned into MCSA bicistronic vector pIRES (Clontech). The wildtype (wt) human $G G C X(h G G C X)$ cDNA was cloned into MCSB of the bicistronic vector. $G G C X$ variants (mut) were introduced by site directed mutagenesis. The reference number of GGCX DNA and protein sequence were RefSeq NM_000821.7 and NP_000821.2, respectively. The $G G C X$ variants were validated by VariantValidator online tool (https://variantvalidator.org/; accessed on 09.04.2021).

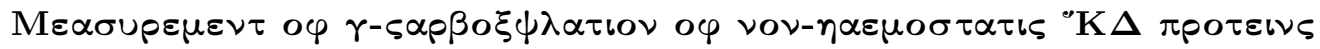

The GGCX wt/mut together with VKD proteins in a bicistronic vector were expressed in a $G G C X^{-/}$ HEK293T cell line as previously described (Ghosh et al., 2021). Four hours post transfection cells were treated with $\mathrm{K}_{1}$. Cell medium and lysate were collected after 48 hours of transfection. Medium was also collected from untransfected cells which served as a negative control. Cell medium and lysates were used to measure $\gamma$-carboxylation by a sandwich ELISA. 96-well plates were coated with anti-myc antibody (Applied biological materials, G077) and were incubated overnight at $4^{\circ} \mathrm{C}$. Wells were washed $3 \mathrm{x}$ with wash buffer $(1 \mathrm{x}$ PBS, pH 7.4, $3 \mathrm{mM} \mathrm{MgCl}_{2}, 0.05 \%$ Tween 20) and blocked in 2\% BSA for 2 hours and incubated overnight with samples in 1:6 dilution. This was followed by $3 \mathrm{x}$ washing and 3 hours incubation with anti-Gla antibody. For MGP we used a MGP-specific Gla antibody (MaGla G8A.1 lot3, gift from Prof. Leon Schurgers). Wells were then washed 3x and incubated with HRP-conjugated antibody (Dako, P0260) for 1 hour at RT followed by HRP-dependent chemiluminescence substrate (Roche, 11582950001) addition and luminescence was detected by a plate reader (Synergy 2, Biotek).

\section{Immunofluorescence staining}

$G G C X^{\text {-/ }}$ cells were seeded on gelatin coated cover-slips and transfected with vectors containing $G G C X$ $w t / m u t$. After 24 hours of transfection cells were washed with PBS, fixed with $4 \%$ paraformaldehyde for 10 minutes and then blocked for 30 minutes with 10\% FBS. Cells were incubated overnight with primary antibodies against GGCX (Abcam, ab170921) and against PDI for ER staining (ThermoFisher, MA3-019). Secondary Alexa Fluor 488 or 594-conjugated antibodies were incubated for 1 hour at RT in dark. Mounting was performed with ProLong Glass (ThermoFisher) including NucBlue. Images were taken by Apotome2 (Zeiss) and Zen software was used to calculated Pearson's correlation coefficient (PCC).

\section{Generation of human GGCX structural model and in silicodocking of vitamin K}

Due to the absence of biophysical structure or adequate homologous structural templates, a hGGCX model was generated on the I-TASSER threading modeling server (https://zhanglab.ccmb.med.umich.edu/ITASSER/) (Roy, Kucukural, \& Zhang, 2010) by submitting the GGCX protein sequence (NP_000812.2) under default conditions. The model1 with the best C-score was used for further analysis but its N/C termini had the same orientation. Since this N/C-terminal orientation of GGCX contradicts previous findings (Tie, Wu, Jin, Nicchitta, \& Stafford, 2000), where opposite orientations have been proposed, N-terminal amino acid residues 1-120 were re-modeled separately in ITASSER and manually replaced onto the previous model. A membrane embedded simulation was performed on the final re-modeled structure in a phosphatidylcholine bilayer in order to equilibrate the model using md_memsim macro embedded in YASARA. The equilibrated model was then docked with $\mathrm{K}_{1}$ hydroquinone downloaded from Pubchem (PUBCHEM ID: 5280585) using the AutoDock module embedded as a macro in YASARA. The top docking poses were analyzed and one docking pose was selected as the final complex based on rational elimination. The selected docked complex was further subjected to membrane embedded simulation for a total of $250 \mathrm{~ns}$ including equilibration and production phase (>100ns). Selected variants S300F and M174R were introduced into the equilibrated docked complex model and both the mutated complexes were simulated in parallel for $>100 \mathrm{~ns}$ in order to understand the structural effect of the variants on GGCX. All simulations, visual rendering and structural analysis were performed on the YASARA version 18.2.24 platform (Land \& Humble, 2018) (Figure S1).

\section{Results}

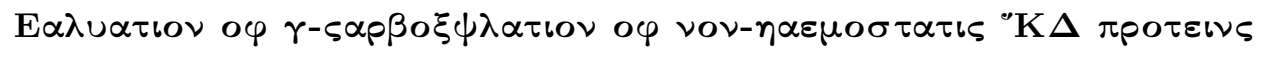

We have analysed the effect of 22 reported GGCX variants on $\gamma$-carboxylation of six non-haemostatic VKD 
proteins. Three proteins i.e. GRP, MGP, and BGLAP were included in our study known to function as calcification regulators. PRGP1 and TMG4 were included as representing transmembrane proteins with unknown function. GAS6 has function in cell signalling (Figure 1). In the following sections, markedly reduced levels of $\gamma$-carboxylated VKD proteins were correlated with the reported patient's phenotypes including skin hyperlaxity, facial dysmorphologies and cardiac abnormalities.

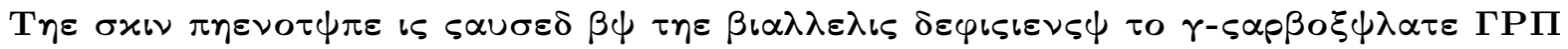

Six VKCFD1 patients were reported with a severe PXE-like phenotype with skin hyper-laxity and skin folds (Goldsmith, Pence, Ratnoff, Adelstein, \& Furie, 1982; Li, Grange et al., 2009; Li, Schurgers et al., 2009; Vanakker et al., 2007). Out of these, four patients were found to be compound heterozygous, where one allele has a loss-of-function or a nonsense variant. This results into monoallelic disability to $\gamma$-carboxylate VKD proteins. The other alleles harbor variants GGCX:p.(R83W), GGCX:p.(V255M), GGCX:p.(G537A) or GGCX:p.(G558R) that exhibited levels between $18-32 \%$ of $\gamma$-carboxylated GRP at $10 \mu \mathrm{M} \mathrm{K}_{1}$ when compared to wt in our assay (Figure 2a, Table 1). Patients harboring variants with greater ability to $\gamma$ carboxylate GRP $>32 \%$ did not show severe skin folds. Levels of $\gamma$-carboxylated MGP varied between 2.9 - $102 \%$ for all these variants (Figure 2a, Table 1). BGLAP, PRGP1, TMG4, and GAS6 are not likely to be causative proteins since all types of dose-responses were detected (Table S1).

In addition, a mild skin phenotype with broadened and agglutinated bundles of fragmented elastic fibres at the age of 47 years was diagnosed in a patient harboring variants GGCX:p.(H404P);p.(R485P) (Watzka et al., 2014). Levels of $\gamma$-carboxylated GRP for these variants were reduced to $35 \%$ and $58 \%$, respectively indicating that patients with greater ability to $\gamma$-carboxylate GRP might exhibit a mild skin phenotype (Figure 2b, Table 1). Levels of $\gamma$-carboxylated MGP are $9 \%$ and $87 \%$, respectively (Figure $2 \mathrm{~b}$, Table 1 ).

Due to low levels of $\gamma$-carboxylated GRP, we are expecting for two more patients with the genotypes GGCX:p.(R83P);p.(R83P) and GGCX:p.(L394R);p.(L394R) the development of a skin phenotype (Figure 2c, Table 1). These patients are currently infants (Brenner et al., 1998; Watzka et al., 2014) and the age of onset was reported to be around 18 years (De Vilder, Eva Y G et al., 2017 Jan 25).

There are two patients, who are heterozygous for GGCX:p.(R476C) or GGCX:p.(R476H), which atypically developed a PXE-like phenotype at the age of 3 or 18 years, respectively (Vanakker et al., 2007). Values of $\gamma$-carboxylated GRP are $42 \%$ or $96 \%$, respectively. Moreover, the other allele in these patients' is wt harboring $100 \%$ activity (Figure S2, Table 1).

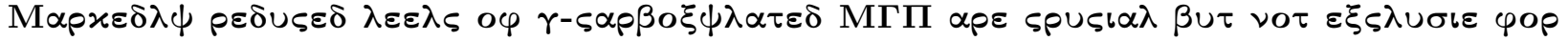

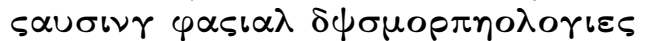

Three VKCFD1 patients were reported with facial dysmorphologies. A Keutel syndrome-like phenotype was described for a patient that is compound heterozygous for GGCX:p.(M174R+R325Q);p.(D153G), where in addition to facial dysmorphologies, strippling of fingers was observed (Tie et al., 2016). We detected for GGCX:p.(D153G) substantially reduced levels of $\gamma$-carboxylated MGP of $38 \%$. Levels of $\gamma$-carboxylated GRP and BGLAP were $94 \%$ and 82\%, respectively. GGCX:p.(M174R) resulted into loss-of-function for all three VKD proteins (Figure 3a, Table 2).

Another patient that was diagnosed with midfacial hypoplasia is homozygous for GGCX:p.(R83P). This pathogenic variant showed markedly reduced levels of $\gamma$-carboxylated MGP, GRP, and BGLAP with 27\%, $13 \%$, and 34\%, respectively (Figure 3a, Table 2). Moreover, this patient is homozygous for the VKORC1:c.1639 AA genotype, which corresponds to a significantly reduced ability of vitamin K recycling.

There is one patient having a midfacial hypoplasia (GGCX:p.(S284P);p.(W315X)), where the allele harboring the nonsense variant leads to monoallelic absence of GGCX protein. The other allele harboring GGCX:p.(S284P) is able to obtain same level of $\gamma$-carboxylated MGP as wt at high $\mathrm{K}$ doses. However, GGCX:p.(S284P) shows significantly reduced ability to $\gamma$-carboxylate MGP at low K concentrations (Figure 3 b, Table 2). Notably, the mother reported a severe hyperemesis gravidarum with a weight loss of seven 
$\mathrm{kg}$ within the first trimester (Watzka et al., 2014). Interestingly, this patient is also homozygous for the VKORC1:c.-1639 AA genotype.

All types of dose-responses were detected for the above mentioned variants for PRGP1, TMG4, and GAS6 (Table S1).

Our results demonstrate that substantial reductions of $\gamma$-carboxylated MGP are associated with facial dysmorphologies in VKCFD1 patients (Figure 3a, Table 2).

Notably, not all VKCFD1 patients with severely reduced levels of $\gamma$-carboxylated MGP as patients harboring GGCX:p.(G558R);p.(F299S), GGCX:p.(V255M);p.(S300F), or GGCX:p.(R83W);p.(Q374X) have skeletal malformation at birth (Figure 3c, Table 2).

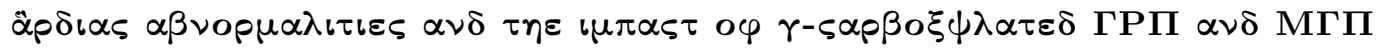

Three patients with $G G C X$ variants p.(R83P);p.(R83P), p.(S284P);p.(W315X), and p.(W157R);p.(T591K+D31N) have a congenital atrial septal defect. Another four patients with variants GGCX:p.(V255M);p.(S300F), GGCX:p.(H404P);p(R485P), GGCX:p.(R476C) and GGCX:p.(G537A);p.(Q374X) developed subclinical atherosclerosis. All these patients harbor pathogenic variants, which show reduced ability to $\gamma$-carboxylate GRP and MGP (Table S3).

All types of dose-responses were detected for BGLAP, GAS6, PRGP1, and TMG4 (Table S1).

\section{hGGCX structural model characteristics}

In order to understand the specific structural implication of the reported pathogenic variants a threading based GGCX in silicomodel was generated. In the simulation-equilibrated model, the majority of the Cterminal region from 420-758aa localized towards the ER luminal side including the propeptide binding site (495-513aa) (Parker et al., 2014) (Figure S3). Our model has multiple transmembrane domains with a distribution of both proper helices (116-134, 240-262, 274-292, 362-377aa) and molten globule regions (95-115, 394-419aa) resembling loosely packed helical structure, which also bears the glutamate binding site (393-404aa) (Figure S3) (Mutucumarana et al., 2000; Mutucumarana, Acher, Straight, Jin, \& Stafford, 2003). In the absence of adequate structural templates and considering the large size of GGCX, our in silico model, albeit entirely hypothetical reflects the best possible structural representation of GGCX protein that can be achieved presently. Reported $G G C X$ variants are heterogeneously distributed throughout thisin silico model (Figure S4).

\section{Identification of the Vitamin K Hydroquinone binding site in GGCX}

Since no knowledge exists regarding $\mathrm{KH}_{2}$ binding, we docked $\mathrm{KH}_{2}$ ligand on our simulation equilibrated model that revealed 29 docking poses (Figure S5). Out of these, only the docking pose 6 had the correct orientation of $\mathrm{KH}_{2}$ (with respect to membrane embedding) and is proximal to known active site residue K218 (Rishavy et al., 2006). Therefore, this docking pose was chosen for further analysis, in which residue F299 undergoes both Pi-Pi and hydrophobic interaction with the $\mathrm{KH}_{2}$ head group (Figure 4a,b and Figure S6). Hence, the substitution of F299 with a polar serine residue can be expected to disrupt its interactions with $\mathrm{KH}_{2}$, resulting into loss-of-function (Figure 4f). To evaluate the effect of GGCX:p.(S300F) and the loss-of-function variant GGCX:p.(M174R) on GGCX structure and $\mathrm{KH}_{2}$ binding, variant models were simulated. The production phase of S300F mutated complex's simulation showed higher root mean square deviation (RMSD) for receptor and ligand movement when compared to wt (Figure 4c). The substitution to phenylalanine breaks a small helix between residues 299-300, disturbing the spatial orientation and Pi-Pi interaction of F299 to the $\mathrm{KH}_{2}$ head group, thereby leading to markedly decreased values of $\gamma$-carboxylation for all investigated VKD proteins. The production phase for the simulation of the M174R mutated docked complex also showed an increase in RMSD, although not as high as S300F (Figure 4c). We assume that M174R most likely alters the structural stability of GGCX which is in line with previous findings (Tie et al., 2016), than interfering with $\mathrm{KH}_{2}$ binding. Further immunofluorescent (IF) staining confirmed that GGCX:p.(F299S) and GGCX:p.(S300F) are co-localizing to the ER similar to wt GGCX with a Pearson's 
correlation coefficient of +0.74 and +0.76 , respectively (Figure 4d,e). GGCX:p.(M174R) co-localizes to the ER with a significantly reduced Pearson's correlation coefficient of +0.53 when compared to wt (Figure 4e). The nonsense variant GGCX:p.(W315X) served as negative control and showed no expression. The positive control GGCX:p.(K218A), where the active site is mutated results into loss-of-function, but is expressed as high as wt and co-localizes in the ER (Figure 4e and Figure S7).

\section{Discussion}

Here, we have identified that undercarboxylated GRP and MGP are the major VKD proteins causing the PXE-like phenotype and facial dysmorphologies in VKCFD1 patients. Individualized medication with $\gamma$ carboxylated VKD proteins to treat non-haemorrhagic phenotypes may represent a promising strategy in future. Furthermore, our GGCX in silico model highlights that two loss-of-function variants affect the $\mathrm{KH}_{2}$ binding pocket in GGCX that was identified in the present study.

The non-haemostatic phenotypes in VKCFD1 summarizes skin laxity, skeletal and cardiac abnormalities (De Vilder, Eva Y G et al., 2017 Jan 25). However, until now it remained elusive which under-carboxylated VKD protein causes the corresponding phenotype.

Notably, we found that GRP plays a fundamental role in the development of the PXE-like phenotype. According to our data, the PXE-like phenotype is caused by the biallelic deficiency to $\gamma$-carboxylate GRP. Four VKCFD1 patients were reported with a severe PXE-like phenotype, where we detected levels of $\gamma$ carboxylated GRP between $0-32 \%$ only by the corresponding pathogenic variants. The monoallelic disability to $\gamma$-carboxylate GRP is not leading to a skin phenotype as we see for all heterozygous family members (parents and siblings) of patients with a PXE-like phenotype (Table S2). These findings suggests that only the biallelic deficiency to $\gamma$-carboxylate GRP lead to a PXE-like phenotype, and brings us to the conclusion that the two patients being heterozygous for either GGCX:p.(R476C) or GGCX:p.(R476H) must have an additional defect because of the unusual early age of onset. Since the reported genotype is also not fitting to the haemorrhagic phenotype as already observed in a previous study (Ghosh et al., 2021), we recommend performing next generation sequencing to potentially identify additional variants in $G G C X$ or other genes.

Due to the markedly reduced levels of $\gamma$-carboxylated GRP obtained in our assay, we predict for two more patients the development of a PXE-like phenotype later in life (Figure 2c). Unfortunately, there is currently no treatment available because life-long administration of high doses of $\mathrm{K}$ will not lead to sufficient $\gamma$ carboxylated levels of GRP as we see in our data. However, these patients might be medicated in future by the nanotechnology-based method of Viegas et al., where human $\gamma$-carboxylated GRP is loaded into extracellular vesicles (Viegas et al., 2019). This recent and smart approach was invented to inhibit vascular calcification, which might have a high potential to treat ectopic calcification of skin, too.

There is a low number of in total three affected patients with facial dysmorphologies. Since loss-offunction variants in $M G P$ lead to Keutel syndrome, which is an autosomal recessive disorder characterized by midfacial hypoplasia and other skeletal malformations (Munroe et al., 1999), it was expected that $G G C X$ variants failing to $\gamma$-carboxylate exclusively MGP lead to midfacial hypoplasia. Tie et al. stated that MGP is the major protein responsible for the Keutel syndrome-like phenotype in a patient carrying GGCX:p.(M174R+R325Q);p.(D153G) (Tie et al., 2016). We confirmed their findings with zero levels of $\gamma$-carboxylated MGP for GGCX:p.(M174R) and with levels of $\sim 38 \%$ for GGCX:p.(D153G) in the other allele. In line with these findings, low levels of $\gamma$-carboxylated MGP below $27 \%$ were detected for the second affected patient with the genotype GGCX:p.(R83P);p.(R83P). However, we even observed lower levels of $\gamma$-carboxylated MGP below $26 \%$ for three other patients that do not have skeletal malformations (GGCX:p.(R83W);p.(Q374X), GGCX:p.(F299S);p.(G558R), and GGCX:p.(V255M);p.(S300F)) (Table 2). Therefore, we conclude that biallelic markedly reduced levels of $\gamma$-carboxylated MGP are crucial but not exclusive for causing the skeletal syndrome in VKCFD1 patients. Since the skeletal phenotype is a congenital defect that is developed during embryogenesis, we suspect that the maternal genotype and vitamin $\mathrm{K}$ uptake during pregnancy contributes to the development of facial malformations as well. Indeed, nutritional uptake by the mother can affect the degree of malformation, which was shown by the study of Lanham et al., 
where $\mathrm{Mgp}^{-/-}$mice, whose mothers were fed on a high-fat diet during pregnancy showed increased bone parameters as bone length, volume, and surface compared to littermates, whose mothers were fed on a control diet. This suggests a maternal nutrient transfer that is influencing the outcome of the birth defects in $\mathrm{Mgp}^{-/}$ mice. Moreover, Lanham et al. also showed that GGCX expression is higher in $\mathrm{Mgp}^{-/-}$mice, whose mothers were fed on high-fat diet when compared to control littermates (Lanham, Cagampang, \& Oreffo, 2018) suggesting that GGCX expression could be regulated through substrate availability. All together this indicates that the nutritional uptake of vitamin $\mathrm{K}$ during pregnancy might modulate the severity of those birth defects in humans as well. Therefore, we assume that the severe hyperemesis gravidarum with a weight loss of seven $\mathrm{kg}$ within the first trimester from the mother of the patient carrying GGCX:p.(S284P);p.(W315X) had a significant additional influence on the development of the patient's midfacial hypoplasia. Although this patient carries one functional allele, where GGCX:p.(S284P) exhibit almost normal level of $\gamma$-carboxylated MGP at high vitamin K levels, this patient was born with midfacial hypoplasia.

Furthermore, we agree that the promoter polymorphism of VKORC1 might modulate the severity of VKCFD1 phenotypes, especially with respect to skeletal malformations (Watzka et al., 2014). In fact, two of the three patients with facial hypoplasia are homozygous for the VKORC1:c.-1639 AA genotype that is associated with $50 \%$ reduction of VKORC1 mRNA and enzymatic activity for each allele (Rieder et al., 2005). The VKORC1 promoter genotype for the third patient is unknown. Although this polymorphism has no impact on haemostasis within normal population, it could influence severity of both haemorrhagic and non-haemorrhagic phenotypes in VKCFD1 patients due to the reduced capacity to recycle vitamin K. Therefore, we recommend to screen VKCFD1 patients also for the VKORC1:c.-1639 genotype in future to evaluate efficiency of therapy. Patients with the weak AA genotype might develop more severe phenotypes and thus need higher $\mathrm{K}$ treatment.

In VKCFD1 patients with a cardiac phenotype, we observed for the corresponding reported pathogenic $G G C X$ variants that the ability to $\gamma$-carboxylate GRP and MGP is reduced (Table S3). However, not all patients carrying pathogenic $G G C X$ variants causing reduced level of $\gamma$-carboxylated GRP and MGP develop cardiac defects. Here, we assume again that the nutritional uptake of vitamin $\mathrm{K}$ and the lifestyle affect the aging phenotype as athereosclerosis, where patients with low vitamin $\mathrm{K}$ uptake have a higher risk to develop a cardiac phenotype.

With respect to the structure of GGCX our model allowed to predict the vitamin K binding site. Two pathogenic variants directly affecting this binding site (GGCX:p.(F299S) and GGCX:p.(S300F)) resulted into loss-of-function of all analysed VKD proteins and were also found in another study to cause loss-offunction or diminished ability to $\gamma$-carboxylate VKD clotting factors (Hao et al., 2020). Hao et al. showed also that these two residues have reduced vitamin $\mathrm{k}$ epoxidation. Although it is only an in silico model of GGCX and it has limitations due to its unique structure, we assume that vitamin K binding takes place in this region. However, future studies are required to solve the X-ray crystal structures of GGCX for identifying the exact vitamin $\mathrm{K}$ binding domain.

Even though, non-haemostatic VKCFD1 phenotypes are rare, this study highlights the need of GGCX to sufficiently $\gamma$-carboxylate GRP and MGP for maintaining physiological calcification. Our data will help to further understand the diversity of known and potentially unknown VKCFD1 phenotypes. The outcome of birth defects could be potentially improved when mothers with a GGCX defect are administered with vitamin $\mathrm{K}$ during pregnancy. Personalized medication with $\gamma$-carboxylated VKD proteins as f.e. $\gamma$-carboxylated GRP to treat non-haemostatic phenotypes may represent a promising strategy in future.

\section{Data Availability}

All $\gamma$-carboxylation data was obtained from triplicate measurements of $n=3$ experiments and is summarized in tables 1 and 2. For figures 1-3 $\gamma$-carboxylation values are given in the supplementary data. If further data is required, data is available on request.

\section{Acknowledgements}


This work was supported, in part, by funding from the Deutsche Forschungsgemeinschaft (DFG) grant (MW and JO; grant no "OL-100-6-1"), Emmy-Noether grant from the DFG (KC, grant no "405008087"), and an investigator-initiated grant provided by Baxalta US Inc., Bannockburn, IL (IIR-DEU-001553), a member of the Takeda group of companies (to JO and MW).

\section{Authorship Contributions}

Experimental design (KJC, JM, SG), generation of GGCX-/- cells (VH, KH, KJC) data collection (SG, $\mathrm{KK}$ ), data analysis \& figure production ( $\mathrm{SG}, \mathrm{KJC}$ ), protein modelling ( $\mathrm{SG}, \mathrm{AB}, \mathrm{FF}$ ) manuscript drafting and editing (SG, KJC, HS, VH, KH, MW, JO).

\section{Conflict of Interest}

The authors declare that there are no conflicts of interest.

\section{Ethical approval statement}

The study does not involve human or animal subjects.

\section{List of Abbreviations}

GGCX, $\gamma$-glutamyl carboxylase; VKCFD, combined deficiency of vitamin K dependent clotting factors; VKD, vitamin $\mathrm{K}$ dependent; VKOR, vitamin K 2,3-epoxide reductase; VKORC1, vitamin K 2,3-epoxide reductase complex subunit 1; wt, wild-type; mut, mutant; PDI, protein disulphide isomerise; BGLAP, bone $\gamma$-carboxyglutamate protein; MGP, matrix Gla protein; UCMA, upper zone of growth plate and cartilage matrix associated protein; GAS6, growth arrest specific 6; PRGP1-2, proline rich and Gla domain 1-2; TMG3-4, transmembrane Gla protein, TM; aa, amino acid.

Table and Figures legends

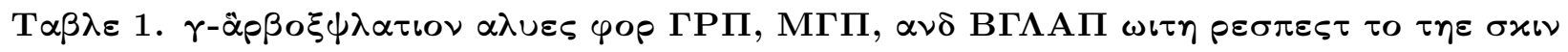
$\pi \eta \varepsilon \nu 0 \tau \psi \pi \varepsilon$

This table summarizes VKCFD1 patients with skin symptoms. For patients with a PXE-like phenotype a biallelic reduction of GRP was detected (highlighted in red). Young VKCFD1 patients with markedly reduced levels of $\gamma$-carboxylated GRP are expected to develop a PXE-like phenotype later in life (highlighted in blue). Pathogenic variants reported in patients with atypical genotype due to the presence of a WT allele, early age of onset, and slightly decreased levels of GRP $\gamma$-carboxylation only (highlighted in Magenta). -, Not analyzed

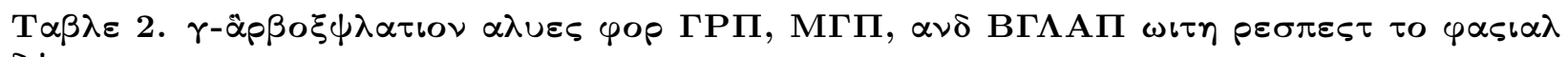
$\delta \psi \sigma \mu о \rho \pi \eta \iota \sigma \mu \varsigma$

This table summarizes the three VKCFD1 patients with facial dysmorphisms. For these patients reduced values of $\gamma$-carboxylated MGP were detected. This table also includes patients with markedly reduced $\gamma$ carboxylated MGP levels although they were not reported with facial dysmorphologies (in black). -, Not analyzed

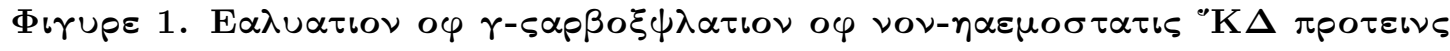

(a) Under normal physiological condition, GGCX $\gamma$-carboxylates specific Glu residues located in VKD proteins to Gla residues rendering them active. VKD proteins which undergoes $\gamma$-carboxylation includes both haemostatic (FII, FVII, FIX, FX, PC, PS and PZ) and non-haemostatic proteins (GRP, MGP, BGLAP, GAS6, PRGPs, and TMGs). (b) Evaluation of $\gamma$-carboxylation for VKD non-haemostatic proteins (GRP, MGP, BGLAP, PRGP1, TMG4, and GAS6) by GGCX wt with a functional ELISA detecting GLA residues. In the dose-response curves the y-axis represents normalized $\gamma$-carboxylation (\%) and the $\mathrm{x}$-axis represents K1 concentrations $[0.1-100 \mu \mathrm{M}]$. (c) Pathological variants in $G G C X$ lead to uncarboxylated or partially $\gamma$-carboxylated VKD proteins, which are inactive. Patients harboring these $G G C X$ variants develop a rare 
bleeding disorder called VKCFD1. Several VKCFD1 patients are diagnosed with non-haemorrhagic phenotype f.e. PXE-like phenotype, facial dysmorphologies and/or cardiac abnormalities. Until now the potential non-haemostatic VKD protein/s responsible for developing these non-haemorrhagic pathological conditions is unknown.

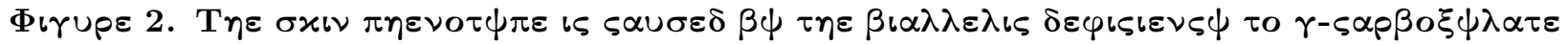 ГРП}

(a-c) $\gamma$-Carboxylation dose-response curves of GRP and MGP measured by ELISA. The y-axis represents normalized $\gamma$-carboxylation (\%) to wt. The $\mathrm{x}$-axis represents $\mathrm{K}_{1}$ concentrations $[0.1-100 \mu \mathrm{M}]$. Error bars depict SD of triplicate measurements of $n=3$ experiments. In each figure dose-response curve for $G G C X$ wt is depicted in black. (a) $\gamma$-Carboxylation values of GRP and MGP with respect to pathogenic $G G C X$ variant reported in patients having a PXE-like phenotype. These variants were found to be compound heterozygous, where the other allele harbors a nonsense or loss-of-function variant. Compound heterozygous pathogenic variants are represented by the same color with each allele variant represented in solid and dotted line. (b) $\gamma$-Carboxylation dose-response curves of GRP and MGP with respect to variants reported to be compound heterozygous in a patient with a mild skin phenotype. (c) $\gamma$-Carboxylation dose-response curves of GRP and MGP with respect to variants that did not respond well to $\mathrm{K}_{1}$ administration. Patients harboring these pathogenic variant are expected to develop a skin phenotype later in life.

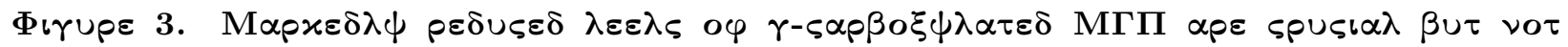

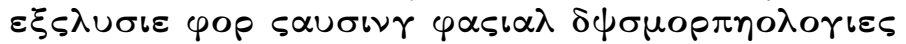

(a-c) $\gamma$-Carboxylation dose-response curves of GRP, MGP, and BGLAP measured by ELISA. The y-axis represents normalized $\gamma$-carboxylation (\%) to wt. The $\mathrm{x}$-axis represents $\mathrm{K}_{1}$ concentrations [0.1-100 $\left.\mu \mathrm{M}\right]$. In each figure dose-response curve for GGCX wt is depicted in black. Compound heterozygous pathogenic variants in one particular patient are represented by the same color with each allele variant in solid and dotted line. Error bars depict SD of triplicate measurements of $n=3$ experiments. (a) $\gamma$-Carboxylation doseresponse curves with respect to variant found in patients having facial dysmorphologies. (b) $\gamma$-Carboxylation dose-responses for a variant reported in a patient having facial dysmorphologies, whose mother had severe hyperemesis gravidarum. The second allele harbors a nonsense variant. (c) Patients with low $\gamma$-carboxylated MGP values that did not developed facial dysmorphologies.

\section{Figure 4. Identification of the Vitamin K Hydroquinone binding site in GGCX}

(a) A snapshot of the GGCX structural model (grey) embedded in membrane (green) with bound $\mathrm{KH}_{2}$ (brown). The model is depicted in ribbon format, whereas the membrane is depicted by its molecular surface. $\mathrm{KH}_{2}$ is depicted in stick format. (b) Close up view of the equilibrated docking pose 6 (brown) with ten interacting residues (blue) and critical interacting residue F299 (purple). Interactions by Pi-Pi and hydrophobic binding with the head group of $\mathrm{KH}_{2}$ are depicted by red and green lines, respectively. The GGCX structural model is depicted in ribbon format while $\mathrm{KH}_{2}$ and interacting residues are depicted in stick format. (c) Receptor and ligand movement RMSD (c- $\alpha$ backbone) for GGCX wt (black), M174R (blue), and S300F (red). The y-axis represents RMSD and x-axis represents simulation time. (d) Colocalization images of HEK293T GGCX -/- cells expressing GGCX wt, F299S, M174R, S300F. First panel represents Dapi counter stain followed by PDI stained with Alexa Fluor 488 (green), GGCX stained with Alexa Fluor 594 (red) and a merged picture. The Pearson's correlation co-efficient is given as Rcoloc in the merged picture. (e) The Pearson's correlation coefficients of GGCX loss-of-function variants with PDI. Error bars depict SD of $n=10$ analyzed cells. ${ }^{* * * *} \mathrm{p}<0.001$ (f) $\gamma$-Carboxylation dose-response curves of VKD proteins with respect to pathogenic variant showing loss-of-function (M174R) and variants involved in $\mathrm{KH}_{2}$ binding (F299S and S300F). The x-axis represents $\mathrm{K}_{1}$ concentrations $[0.1-100 \mu \mathrm{M}]$. Error bars represent SD of $n=$ 3 measurements of $n=3$ experiments. 


\section{References}

Berkner, K. L., \& Runge, K. W. (2004). The physiology of vitamin K nutriture and vitamin K-dependent protein function in atherosclerosis. Journal of Thrombosis and Haemostasis : JTH , 2 (12), 2118-2132. https://doi.org/10.1111/j.1538-7836.2004.00968.x

Brenner, B., Sanchez-Vega, B., Wu, S. M., Lanir, N., Stafford, D. W., \& Solera, J. (1998). A missense mutation in gamma-glutamyl carboxylase gene causes combined deficiency of all vitamin K-dependent blood coagulation factors. Blood , 92 (12), 4554-4559.

Darghouth, D., Hallgren, K. W., Shtofman, R. L., Mrad, A., Gharbi, Y., Maherzi, A., . . Rosa, J.-P. (2006). Compound heterozygosity of novel missense mutations in the gamma-glutamyl-carboxylase gene causes hereditary combined vitamin K-dependent coagulation factor deficiency.Blood , 108 (6), 1925-1931. https://doi.org/10.1182/blood-2005-12-010660

De Vilder, Eva Y G, Debacker, J., \& Vanakker, O. M. (2017 Jan 25).Ggcx-Associated Phenotypes: An Overview in Search of Genotype-Phenotype Correlations . Switzerland. https://doi.org/10.3390/ijms18020240

Ghosh, S., Kraus, K., Biswas, A., Müller, J., Buhl, A.-L., Forin, F., . . Oldenburg, J. (2021). Ggcx mutations show different responses to vitamin $\mathrm{K}$ thereby determining the severity of the hemorrhagic phenotype in VKCFD1 patients. Journal of Thrombosis and Haemostasis : JTH.Advance online publication. https://doi.org/10.1111/jth.15238

Goldsmith, G. H., JR, Pence, R. E., Ratnoff, O. D., Adelstein, D. J., \& Furie, B. (1982). Studies on a family with combined functional deficiencies of vitamin K-dependent coagulation factors. The Journal of Clinical Investigation , 69 (6), 1253-1260. https://doi.org/10.1172/jci110564

Hao, Z., Jin, D., Chen, X., Schurgers, L. J., Stafford, D. W., \& Tie, J.-K. (2020). Gamma-glutamyl carboxylase mutations differentially affect the biological function of vitamin K-dependent proteins. Blood.Advance online publication. https://doi.org/10.1182/blood.2020006329

Kulman, J. D., Harris, J. E., Xie, L., \& Davie, E. W. (2001). Identification of two novel transmembrane gamma-carboxyglutamic acid proteins expressed broadly in fetal and adult tissues. Proceedings of the National Academy of Sciences of the United States of America , 98 (4), 1370-1375. https://doi.org/10.1073/pnas.98.4.1370

Kurachi, K., \& Davie, E. W. (1982). Isolation and characterization of a cDNA coding for human factor IX. Proceedings of the National Academy of Sciences of the United States of America, 79 (21), 6461-6464. https://doi.org/10.1073/pnas.79.21.6461

Lacombe, J., \& Ferron, M. (2015). Gamma-carboxylation regulates osteocalcin function. Oncotarget , 6 (24), 19924-19925. https://doi.org/10.18632/oncotarget.5126

Land, H., \& Humble, M. S. (2018). Yasara: A Tool to Obtain Structural Guidance in Biocatalytic Investigations. Methods in Molecular Biology (Clifton, N.J.) , 1685 , 43-67. https://doi.org/10.1007/978-1-49397366-8_4

Lanham, S. A., Cagampang, F. R., \& Oreffo, R. O. C. (2018). The influence of a high fat diet on bone and soft tissue formation in Matrix Gla Protein knockout mice. Scientific Reports , 8 (1), 3635. https://doi.org/10.1038/s41598-018-21650-0

Leytus, S. P., Foster, D. C., Kurachi, K., \& Davie, E. W. (1986). Gene for human factor X: A blood coagulation factor whose gene organization is essentially identical with that of factor IX and protein C.Biochemistry , 25 (18), 5098-5102. https://doi.org/10.1021/bi00366a018

Li, Q., Grange, D. K., Armstrong, N. L., Whelan, A. J., Hurley, M. Y., Rishavy, M. A., . . Uitto, J. (2009). Mutations in the GGCX and ABCC6 genes in a family with pseudoxanthoma elasticum-like phenotypes. The 
Journal of Investigative Dermatology , 129 (3), 553-563. https://doi.org/10.1038/jid.2008.271

Li, Q., Schurgers, L. J., Smith, A. C. M., Tsokos, M., Uitto, J., \& Cowen, E. W. (2009). Co-existent pseudoxanthoma elasticum and vitamin K-dependent coagulation factor deficiency: Compound heterozygosity for mutations in the GGCX gene. The American Journal of Pathology ,174 (2), 534-540. https://doi.org/10.2353/ajpath.2009.080865

Manfioletti, G., Brancolini, C., Avanzi, G., \& Schneider, C. (1993). The protein encoded by a growth arrestspecific gene (gas6) is a new member of the vitamin K-dependent proteins related to protein S, a negative coregulator in the blood coagulation cascade. Molecular and Cellular Biology , 13 (8), 4976-4985. https://doi.org/10.1128/mcb.13.8.4976

Munroe, P. B., Olgunturk, R. O., Fryns, J. P., van Maldergem, L., Ziereisen, F., Yuksel, B., . . . Chung, E. (1999). Mutations in the gene encoding the human matrix Gla protein cause Keutel syndrome. Nature Genetics ,21 (1), 142-144. https://doi.org/10.1038/5102

Mutucumarana, V. P., Stafford, D. W., Stanley, T. B., Jin, D. Y., Solera, J., Brenner, B., . . . Wu, S. M. (2000). Expression and characterization of the naturally occurring mutation L394R in human gamma-glutamyl carboxylase. The Journal of Biological Chemistry , 275 (42), 32572-32577. https://doi.org/10.1074/jbc.M006808200

Mutucumarana, V. P., Acher, F., Straight, D. L., Jin, D.-Y., \& Stafford, D. W. (2003). A conserved region of human vitamin K-dependent carboxylase between residues 393 and 404 is important for its interaction with the glutamate substrate. The Journal of Biological Chemistry ,278 (47), 46488-46493. https://doi.org/10.1074/jbc.M307707200

Nelsestuen, G. L. (1999). Enhancement of vitamin-K-dependent protein function by modification of the gamma-carboxyglutamic acid domain: Studies of protein $\mathrm{C}$ and factor VII. Trends in Cardiovascular Medicine ,9 (6), 162-167. https://doi.org/10.1016/s1050-1738(99)00024-9

Parker, C. H., Morgan, C. R., Rand, K. D., Engen, J. R., Jorgenson, J. W., \& Stafford, D. W. (2014). A conformational investigation of propeptide binding to the integral membrane protein gamma-glutamyl carboxylase using nanodisc hydrogen exchange mass spectrometry. Biochemistry ,53 (9), 1511-1520. https://doi.org/10.1021/bi401536m

Poser, J. W., Esch, F. S., Ling, N. C., \& Price, P. A. (1980). Isolation and sequence of the vitamin Kdependent protein from human bone. Undercarboxylation of the first glutamic acid residue. The Journal of Biological Chemistry, 255 (18), 8685-8691.

Price, P. A., Fraser, J. D., \& Metz-Virca, G. (1987). Molecular cloning of matrix Gla protein: Implications for substrate recognition by the vitamin K-dependent gamma-carboxylase. Proceedings of the National Academy of Sciences of the United States of America , 84 (23), 8335-8339. https://doi.org/10.1073/pnas.84.23.8335

Rieder, M. J., Reiner, A. P., Gage, B. F., Nickerson, D. A., Eby, C. S., McLeod, H. L., . . Rettie, A. E. (2005). Effect of VKORC1 haplotypes on transcriptional regulation and warfarin dose. The New England Journal of Medicine , 352 (22), 2285-2293. https://doi.org/10.1056/NEJMoa044503

Rishavy, M. A., \& Berkner, K. L. (2012). Vitamin K oxygenation, glutamate carboxylation, and processivity: Defining the three critical facets of catalysis by the vitamin K-dependent carboxylase. Advances in Nutrition (Bethesda, Md.) , 3 (2), 135-148. https://doi.org/10.3945/an.111.001719

Rishavy, M. A., Hallgren, K. W., Yakubenko, A. V., Shtofman, R. L., Runge, K. W., \& Berkner, K. L. (2006). Bronsted analysis reveals Lys218 as the carboxylase active site base that deprotonates vitamin $\mathrm{K}$ hydroquinone to initiate vitamin K-dependent protein carboxylation. Biochemistry ,45 (44), 13239-13248. https://doi.org/10.1021/bi0609523

Roy, A., Kucukural, A., \& Zhang, Y. (2010). I-TASSER: A unified platform for automated protein structure and function prediction. Nature Protocols , 5 (4), 725-738. https://doi.org/10.1038/nprot.2010.5 
Spronk, H. M., Farah, R. A., Buchanan, G. R., Vermeer, C., \& Soute, B. A. (2000). Novel mutation in the gamma-glutamyl carboxylase gene resulting in congenital combined deficiency of all vitamin K-dependent blood coagulation factors. Blood , 96 (10), 3650-3652.

Stenflo, J., Fernlund, P., Egan, W., \& Roepstorff, P. (1974). Vitamin K dependent modifications of glutamic acid residues in prothrombin.Proceedings of the National Academy of Sciences of the United States of America , 71 (7), 2730-2733. https://doi.org/10.1073/pnas.71.7.2730

Tie, J., Wu, S. M., Jin, D., Nicchitta, C. V., \& Stafford, D. W. (2000). A topological study of the human gamma-glutamyl carboxylase. Blood ,96 (3), 973-978.

Tie, J.-K., Carneiro, J. D. A., Jin, D.-Y., Martinhago, C. D., Vermeer, C., \& Stafford, D. W. (2016). Characterization of vitamin K-dependent carboxylase mutations that cause bleeding and nonbleeding disorders. Blood , 127 (15), 1847-1855. https://doi.org/10.1182/blood-2015-10-677633

Vanakker, O. M., Martin, L., Gheduzzi, D., Leroy, B. P., Loeys, B. L., Guerci, V. I., . . Paepe, A. de (2007). Pseudoxanthoma elasticum-like phenotype with cutis laxa and multiple coagulation factor deficiency represents a separate genetic entity. The Journal of Investigative Dermatology , 127 (3), 581-587. https://doi.org/10.1038/sj.jid.5700610

Vermeer, C. (1990). Gamma-carboxyglutamate-containing proteins and the vitamin K-dependent carboxylase. The Biochemical Journal , 266 (3), 625-636. https://doi.org/10.1042/bj2660625

Viegas, C., Edelweiss, E., Schneider, J., Schaeffer-Reiss, C., Poterszman, A., Rafael, M., . . . Simes, D. (2019). Use of an innovative system and nanotechnology-based strategy for therapeutic applications of Glarich protein (GRP). Annals of Medicine , 51 (sup1), 38. https://doi.org/10.1080/07853890.2018.1561804

Viegas, C. S. B., Cavaco, S., Neves, P. L., Ferreira, A., Joao, A., Williamson, M. K., . . . Simes, D. C. (2009). Gla-rich protein is a novel vitamin K-dependent protein present in serum that accumulates at sites of pathological calcifications. The American Journal of Pathology ,175 (6), 2288-2298. https://doi.org/10.2353/ajpath.2009.090474

Viegas, C. S. B., Rafael, M. S., Enriquez, J. L., Teixeira, A., Vitorino, R., Luis, I. M., . . Simes, D. C. (2015). Gla-rich protein acts as a calcification inhibitor in the human cardiovascular system.Arteriosclerosis, Thrombosis, and Vascular Biology , 35 (2), 399-408. https://doi.org/10.1161/ATVBAHA.114.304823

Watzka, M., Geisen, C., Scheer, M., Wieland, R., Wiegering, V., Dorner, T., . . . Oldenburg, J. (2014). Bleeding and non-bleeding phenotypes in patients with GGCX gene mutations. Thrombosis Research ,134 (4), 856-865. https://doi.org/10.1016/j.thromres.2014.07.004

Willems, B. A. G., Vermeer, C., Reutelingsperger, C. P. M., \& Schurgers, L. J. (2014). The realm of vitamin K dependent proteins: Shifting from coagulation toward calcification. Molecular Nutrition $\&$ Food Research , 58 (8), 1620-1635. https://doi.org/10.1002/mnfr.201300743

\section{Hosted file}

Table 1.pdf available at https://authorea.com/users/409373/articles/519084-ggcx-variantsleading-to-biallelic-deficiency-to-\%CE\%B3-carboxylate-grp-cause-skin-laxity-in-vkcfd1patients

\section{Hosted file}

Table 2.pdf available at https://authorea.com/users/409373/articles/519084-ggcx-variantsleading-to-biallelic-deficiency-to-\%CE\%B3-carboxylate-grp-cause-skin-laxity-in-vkcfd1patients 
Figure 1

(a)

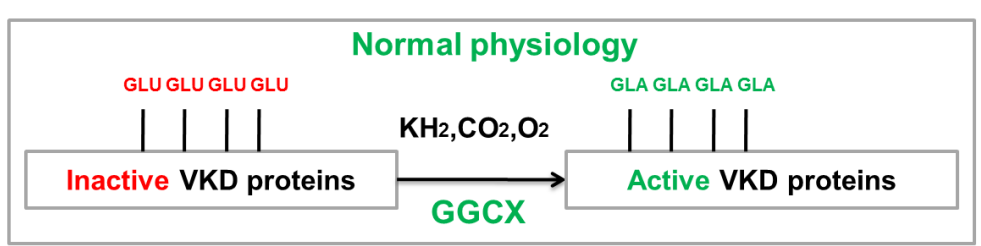

(b)
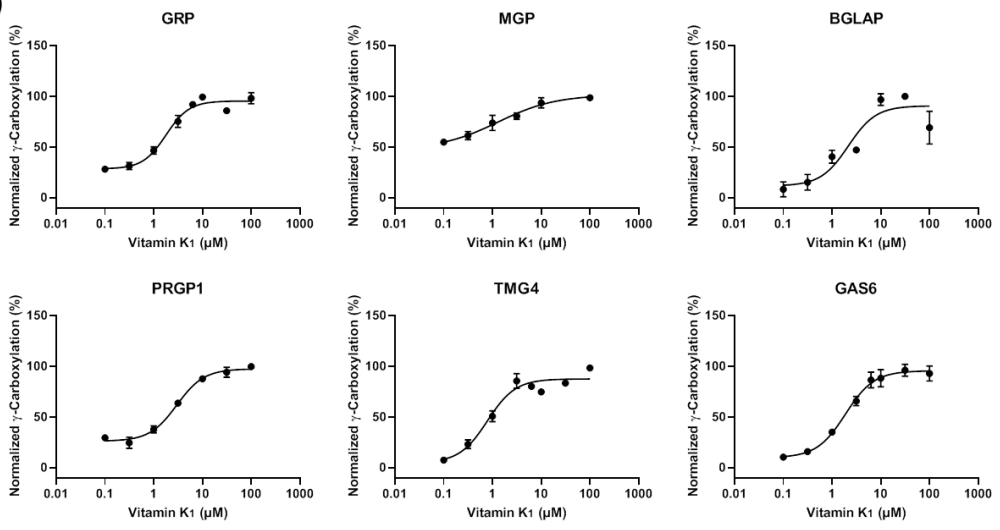

(c)

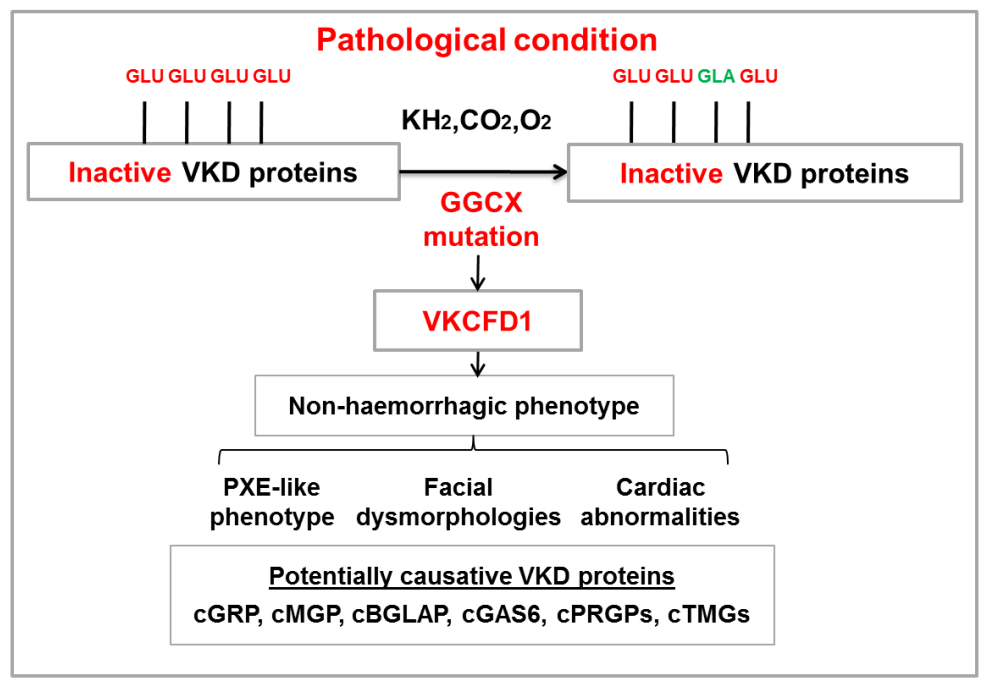


Figure 2

(a)

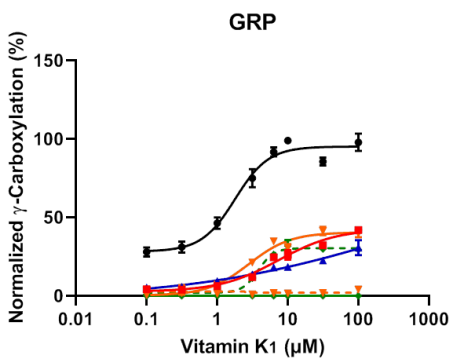

PXE-like phenotype
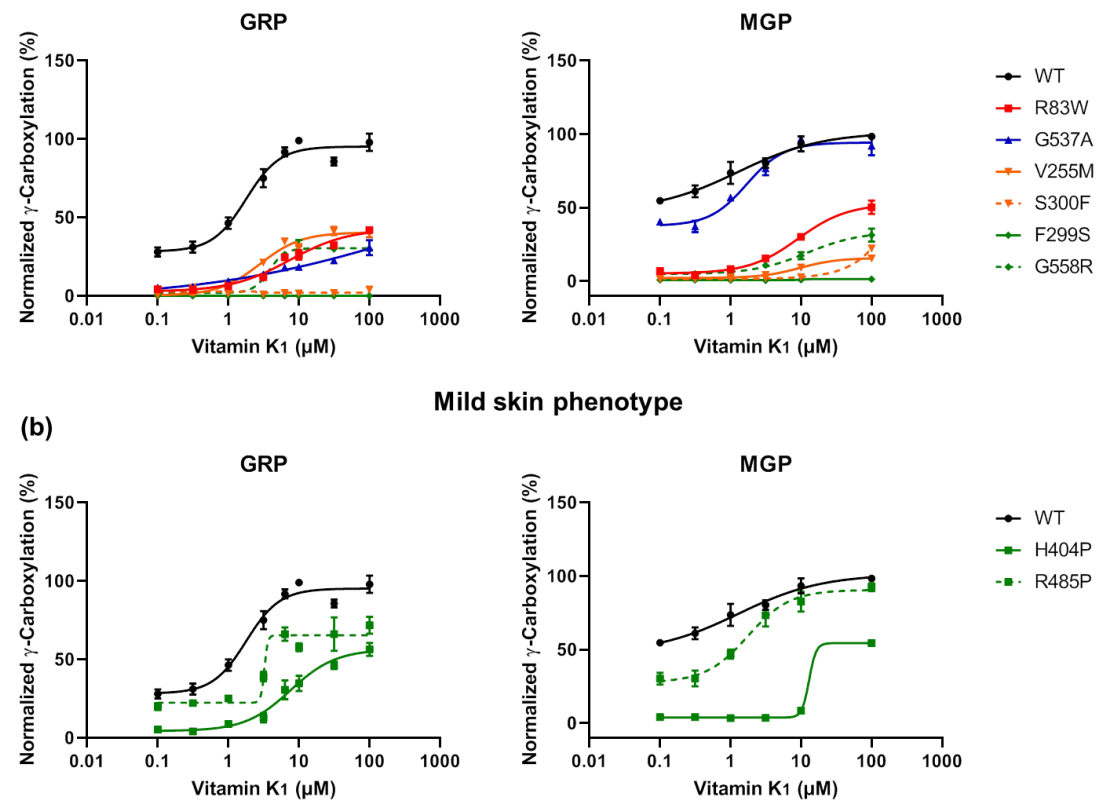

Mild skin phenotype

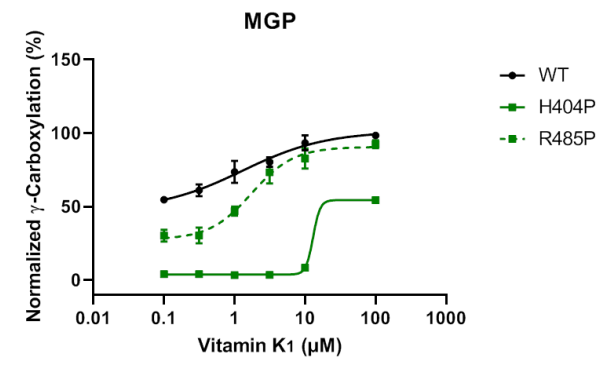

(c)

Expected to develop PXE-like phenotype
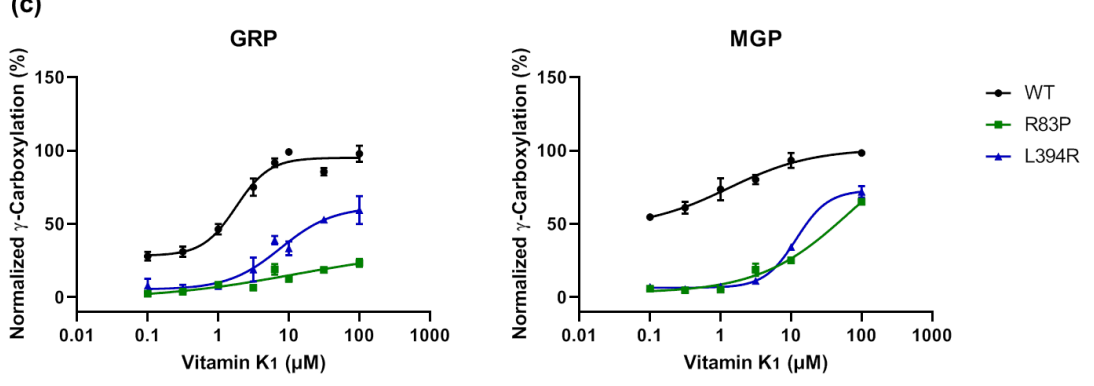

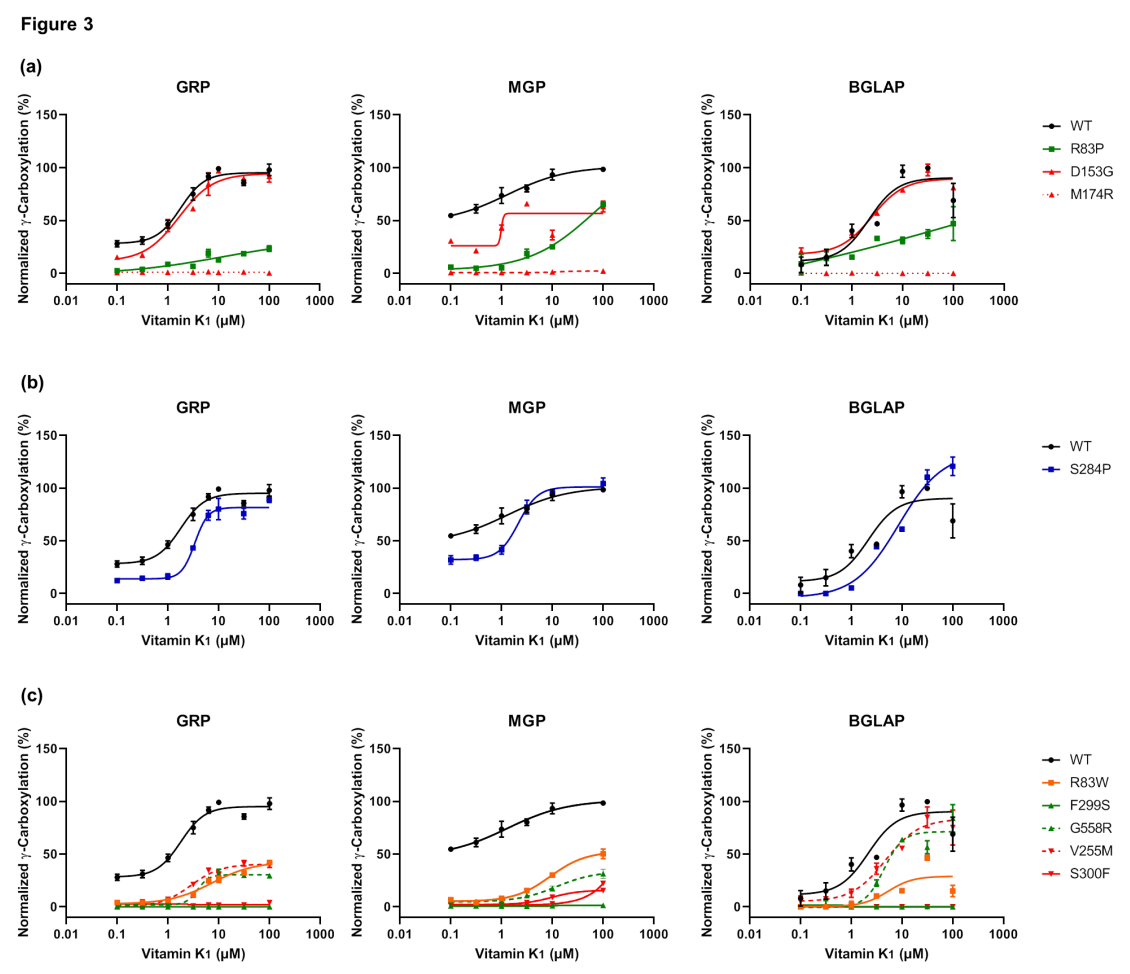

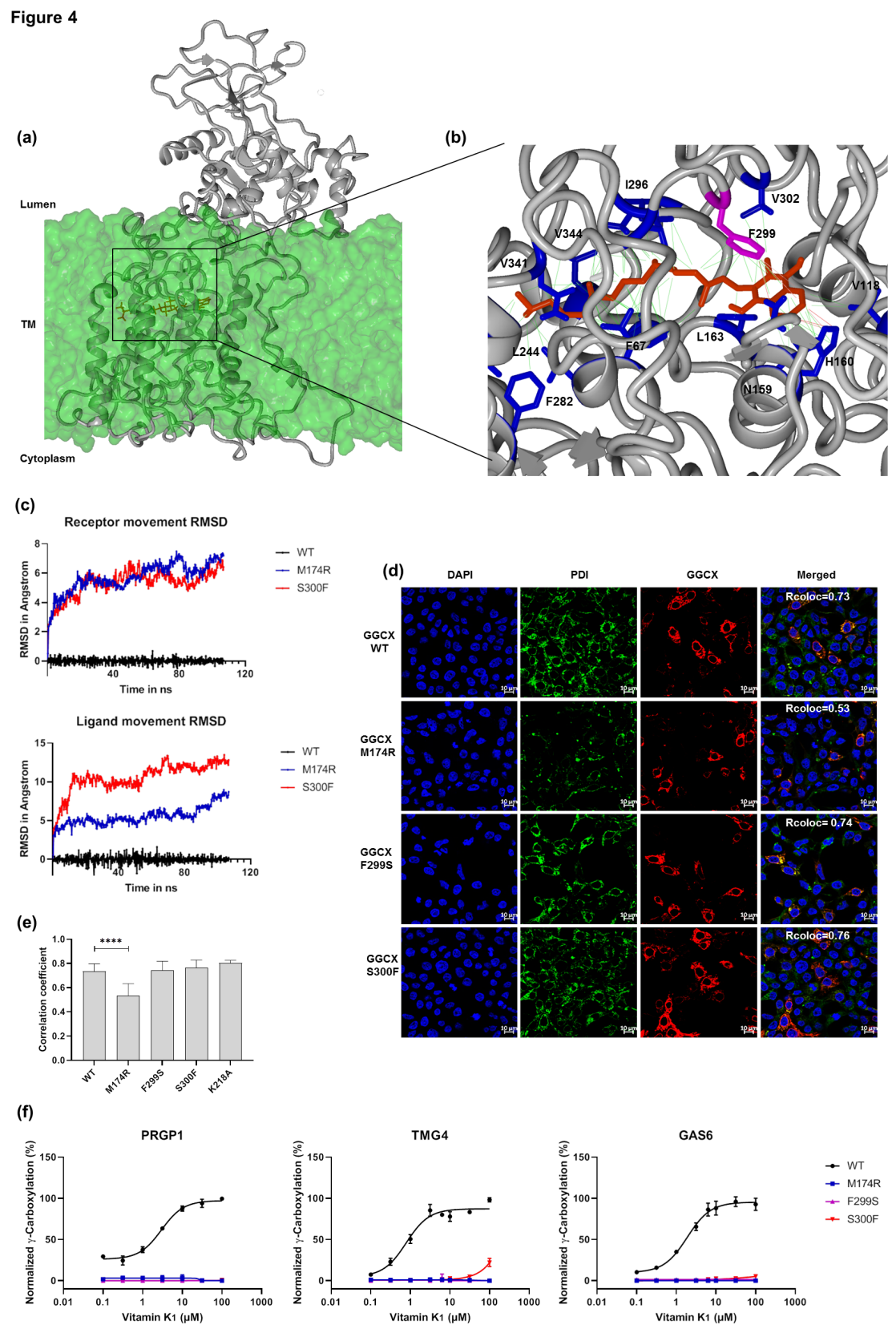